\title{
BMJ Open Is arthroplaSty bEtter than interNal fixation for undiSplaced femoral nEck fracture? A national pragmatic RCT: the SENSE trial
}

\author{
Bjarke Viberg (D , ,,2 Søren Kold, ${ }^{3}$ Ole Brink, ${ }^{4}$ Morten Schultz Larsen, ${ }^{2}$ \\ Kristoffer Borbjerg Hare, ${ }^{5,6,7}$ Henrik Palm, ${ }^{8}$ SENSE collaborators
}

To cite: Viberg B, Kold S, Brink 0, et al. Is arthroplaSty bEtter than interNal fixation for undiSplaced femoral nEck fracture? A national pragmatic RCT: the SENSE trial. BMJ Open 2020;10:e038442. doi:10.1136/ bmjopen-2020-038442

- Prepublication history and additional material for this paper are available online. To view these files, please visit the journal online (http://dx.doi. org/10.1136/bmjopen-2020038442).

Received 10 March 2020 Revised 20 July 2020 Accepted 09 September 2020

Check for updates

(c) Author(s) (or their employer(s)) 2020. Re-use permitted under CC BY-NC. No commercial re-use. See rights and permissions. Published by BMJ.

For numbered affiliations see end of article.

Correspondence to

Dr Bjarke Viberg;

bjarke.viberg@rsyd.dk

\section{ABSTRACT}

Introduction Undisplaced femoral neck fractures

(FNFs) are usually treated by internal fixation (IF)

but two randomised controlled trials (RCTs) have demonstrated advantages of treatment with arthroplasty.

The complication rate was lowered but there were no clinically improved patient-reported outcome measures (PROM), which could be due to underpowering or choice of selected PROM as the studies do appear to report a better functional outcome. We will conduct an RCT comparing IF with arthroplasties in patients aged over 65 years with an undisplaced FNF.

Methods and analysis All hospitals in Denmark treating patients with hip fracture can provide patients for this study; therefore, the study can be considered a national RCT. Patients over 65 years old with an undisplaced FNF will be screened for eligibility and patients will only be excluded if they are unable to understand the study information (due to dementia or language), if they have a posterior tilt $>20^{\circ}$, a pathological fracture or they cannot walk. Participants will be electronically randomised (in alternating blocks of 4 or 6 ) into either IF or arthroplasty. Postoperative care will follow the department standards. Primary and secondary outcomes and measuring points have been established in collaboration with patients with hip fracture by focus group interviews. The primary outcome measure is the New Mobility Score assessed after 1 year. Secondary outcomes are the Oxford Hip Score, EuroQol 5 domain (EQ-5D-5L), degree of posterior tilt, pain Verbal Rating Scale, reoperation and mortality.

Ethics and dissemination The study is approved by the Danish Data Protection Agency (19/7429) and the scientific ethics committee (S-20180036). All participants will sign an informed consent before entering the trial. Because this is a national trial, all relevant healthcare professionals in Denmark will automatically receive the trial results that will be published in international peer-reviewed journals. Trial registration number ClinicalTrials.gov Registry (NCT04075461).

\section{INTRODUCTION}

Arthroplasty for a displaced femoral neck fracture (FNF) in the elderly is recommended by most guidelines. ${ }^{12}$ For the undisplaced FNF, internal fixation is the only recommendation;
Strengths and limitations of this study

- National randomised controlled trial (RCT) on undisplaced femoral neck fracture comparing internal fixation with arthroplasty.

- It is a pragmatic RCT and each hospital can use their preferred implants.

- Primary outcome is an easily understood functional score, the New Mobility Score.

- Participants are only included if they are able to walk outside with no help from other individuals.

- The participants and assessors are blinded concerning implant.

however, it is questioned whether there is an alternative treatment.

Recently, two randomised controlled trials (RCTs) comparing internal fixation with hemiarthroplasty have been published. ${ }^{3}{ }^{4}$ Both RCTs demonstrated a lower reoperation frequency in the hemiarthroplasty group $(5 \%)$ compared with internal fixation (20\%$21 \%$ ). Lu $e t a l^{3}$ found a slightly higher Harris Hip Score after 6 months and 1 year in favour of hemiarthroplasty but not thereafter. Dolatowski $e t a t^{4}$ found a faster mobility (TimedUp-And-Go) but no difference in the Harris Hip Score. These studies did not show a clinical difference in Harris Hip Scores, but this measure may not be the best primary outcome measure due to the ceiling effect and lack of validation for patients with hip fracture. ${ }^{5}$

Mobilisation after hip fracture is perhaps the most important factor for mortality after surgery $^{6}$ and surgery should, therefore, aim for fast mobilisation. Arthroplasty may be a good choice, as it may yield faster recovery than internal fixation. ${ }^{7}$ A systematic review ${ }^{8}$ in 2008 of mobility instruments for older patients showed that no existing instrument had the properties required to measure and monitor the mobility of older acute medical 
patients accurately. The New Mobility Score (NMS) developed by Parker may predict mortality, ${ }^{9}$ and Kristensen et al have since shown that it can also predict function better than Timed-Up-and-Go. NMS is easy to use and has a very high inter-tester reliability. ${ }^{10}{ }^{11}$ Pedersen and Lauritsen ${ }^{12}$ also demonstrated good correlation of NMS and gait function prediction with the same properties as Barthel-20 and Barthel-100 but with a lower ceiling frequency of 4 months postoperatively.

Even though the evidence is limited from the two RCTs, ${ }^{34}$ one could argue for implementing arthroplasty for undisplaced FNF since there are fewer reoperations and perhaps a faster mobilisation. However, a cohort study has demonstrated a higher mortality percentage when using hemiarthroplasty compared with internal fixation. ${ }^{13}$ This study does contain selection bias and confounding problems, as there are in general with cohort studies, which makes the resulting evidence limited for everyday clinical use. Therefore, we should conduct larger RCTs as hip fracture RCTs, in general, are small and underpowered. ${ }^{14}$ In addition, external validity is often a problem in traditional RCTs, because an inclusion rate as little as $7 \%$ was seen in the FAITH Study ${ }^{15}$ thereby questioning whether hip fracture trials exclude too many patients. ${ }^{16}$ A pragmatic RCT design includes a larger proportion of the eligible patients due to fewer exclusion criteria and could, therefore, be a better choice to test an intervention in everyday clinical setting. ${ }^{17}$

The aim of this trial is to compare functional outcomes of arthroplasty with internal fixation for patients over 65 years old with an undisplaced FNF. The study is designed as a national single-blinded pragmatic 1:1 RCT. The hypothesis states that arthroplasty is superior to internal fixation using the NMS as the primary outcome.

\section{METHODS: PARTICIPANTS, INTERVENTIONS AND OUTCOMES Study setting}

The Danish National Health Service provides taxsupported free healthcare and general hospital care for all Danish citizens. ${ }^{18}$ All patients with hip fracture are treated at public hospitals in Denmark as no private hospitals in Denmark have any acute fracture treatment. Twenty public hospitals in Denmark treat patients with hip fracture and all participate in this study, making this trial a national RCT.

The trial started 1st of February 2020 but has been paused due to COVID-19. The sites will start recruitment at different time points from 1st of July to 1st of October.

\section{Trial design}

The study is designed as a national pragmatic RCT, including all patients with an undisplaced FNF and an NMS of 5 and above. The current standard treatment in Denmark is internal fixation, and patients are randomised to either arthroplasty or internal fixation. The steering group has assessed the pragmatic attitude of the design and the study reaches five points in seven of the nine

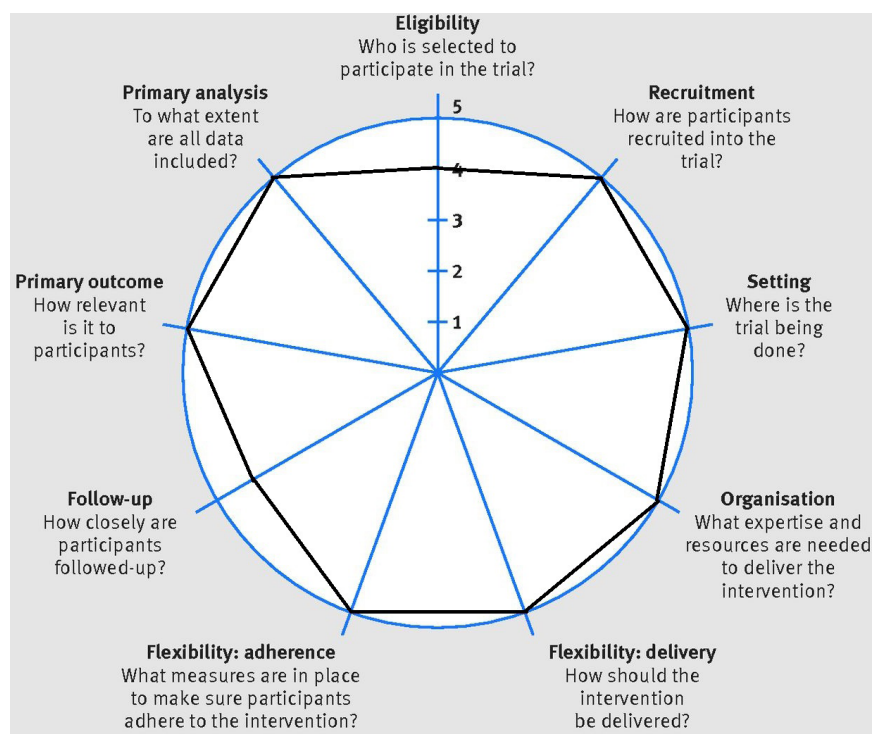

Figure 1 Assessment of pragmatic design using the PRagmatic Explanatory Continuum Indicator Summary-2 wheel.

domains (figure 1). ${ }^{17}$ Reporting will be performed according to the extension of the Consolidated Standards of Reporting Trials statements for a pragmatic RCT, ${ }^{19}$ and this protocol is reported according to the Standard Protocol Items: Recommendations for Interventional Trials statement. $^{20}$

\section{Eligibility criteria}

All patients with an undisplaced FNF classified as either Garden type $\mathrm{I}$ or $\mathrm{II}^{21}$ are evaluated. The patients are included if:

- Age $\geq 65$ years.

- Undisplaced FNF.

- Posterior tilt ${ }^{22}<20^{\circ}$.

- $\mathrm{NMS}=5$ and above, indicating an ability to walk prior to the fracture.

- Cognitive state intact to achieve informed consent. Patients are excluded if:

- The fracture is pathological.

- The patient does not speak or understand Danish language.

To ensure correct fracture classification, an adjudication committee will evaluate all included $\mathrm{X}$-ray images.

\section{Interventions}

Participants are randomised to either arthroplasty or internal fixation. Because the treatment options at each hospital may be very different, arthroplasty can include total hip arthroplasty (cemented, uncemented, hybrid, dual-mobility cup) or hemiarthroplasty (cemented, uncemented) using the institution's regular surgical approach (18 hospitals only use the posterior approach). Internal fixation can include either two or three screws/pins or a sliding hip screw. After discharge, all patients will be referred to standard rehabilitation in the municipalities and will be seen in their own home or at the orthopaedic 
department for outcome assessment after 3, 6 and 12 months. X-ray will be performed postoperatively within discharge and after 12 months.

\section{Outcomes}

Primary and secondary outcomes and measuring points have been established in collaboration with a focus group interview with patients with hip fracture. The primary outcome measure is NMS assessed after 12 months. NMS will also be assessed at baseline, 3 and 6 months.

Secondary outcome measures evaluated at the same time points are the Oxford Hip Score, EuroQol 5 domain, pain Verbal Rating Scale, ${ }^{23}$ reoperation (any surgery related to the implants including closed reduction) and mortality. Explorative outcome measures are the de Morton Mobility Index, ${ }^{74}$ Barthel-20, ${ }^{25}$ Cumulated Ambulation Score, ${ }^{26}$ X-ray measurements and activity tracking.

Information will be retrieved from patient interview and healthcare records on the following:

- Demographics: age, sex, residency, prefracture mobility.

- Comorbidity: American Society of Anaesthesiologists Classification, diseases, medication, smoking, alcohol.

- Admission: time of admission, duration of hospital stay, concurrent infection, fracture time.

- Surgery: start and end of surgery, type of implant, surgical experience, blood loss.

- X-ray: quality of implant positioning. ${ }^{27-29}$

- Biochemistry: haemoglobin, leucocytes, C reactive protein, estimated glomerular filtration rate, international normalised ratio, blood transfusions.
- Complications: postoperative medical complications (all possible such as heart, lung, abdominal, brain, electrolytes, fall and infection), readmissions.

A timeframe for the collection of data is provided in table 1. Healthcare record information is collected for research purposes only to compare patient groups and treatment.

\section{Sample size calculation}

A minimal clinically important difference in NMS of 1 point was taken from Kristensen's thesis ${ }^{30}$ and a 1-year average of 6.4 points with an SD of 2.2 from Steihaug et $a l .{ }^{31}$ The sample size was calculated using a 1-point difference, an SD of 2.2, allowing a 5\% probability of type 1 error and applying 95\% statistical power. Consequently, 127 patients are required in each group and to allow for loss during follow-up due to mortality and other causes $(30 \%)$. Therefore, a total of 330 patients are required for the study.

\section{Recruitment}

All patients are recruited in the emergency department when diagnosed with an undisplaced FNF. The admitting doctor or a senior consultant will inform the patient about the trial while the patient is in the emergency department. The information will be given verbally as well as by written participant information in an undisturbed room in the emergency department. If no next of kin are present, they will be invited to attend by phone if requested by the participant. Otherwise, an impartial assessor can be assigned. Because surgery is required to take place as quickly as possible due to a higher risk of

Table 1 Timeframe for collection of data

\begin{tabular}{|c|c|c|c|c|c|c|c|}
\hline & Admission & 2 weeks & 6 weeks & 3 months & 6 months & 12 months & At event \\
\hline Demographics & $x$ & & & & & & \\
\hline Comorbidity & $\mathrm{x}$ & & & & & & \\
\hline Admission & $x$ & & & & & & \\
\hline Surgery & $x$ & & & & & & \\
\hline Blood & $x$ & & & & & & \\
\hline X-ray & $\mathrm{x}$ & & & & & $x$ & \\
\hline NMS & $x$ & & & $x$ & $x$ & $x$ & \\
\hline Pain VRS & $\mathrm{x}$ & $\mathrm{x}$ & $\mathrm{x}$ & $x$ & $x$ & $x$ & \\
\hline $\mathrm{OHS}$ & $x$ & & & $x$ & $x$ & $x$ & \\
\hline EQ-5D-5L & $\mathrm{x}$ & & & $\mathrm{x}$ & $x$ & $\mathrm{x}$ & \\
\hline DEMMI & $\mathrm{x}$ & & & $\mathrm{x}$ & $x$ & $\mathrm{x}$ & \\
\hline Barthel-20 & $\mathrm{x}$ & & & $x$ & $x$ & $x$ & \\
\hline CAS & $x$ & & $x$ & $x$ & & & \\
\hline Reoperation & & & & & & & $x$ \\
\hline Complications & & & & & & & $x$ \\
\hline Mortality & & & & & & & $x$ \\
\hline
\end{tabular}

CAS, Cumulated Ambulation Score; DEMMI, de Morton Mobility Index; EQ-5D-5L, EuroQol 5 domain; NMS, New Mobility Score; OHS,

Oxford Hip Score; VRS, Verbal Rating Scale. 
mortality when delaying surgery, a reflection time of only 2 hours has been approved. Retrieval of informed consent will take place at either the emergency department or the ward.

\section{Patient and public involvement}

We conducted a focus group interview with patients with hip fracture with internal fixation or arthroplasty at Hospital Lillebaelt, which involved six patients (and their relatives) aged 70-94 years who had received surgery 6-12 months prior to the interview. They were interviewed using a structured interview guide. The interview consisted of open questions regarding their hip fracture experience, their subsequent consequences/challenges and what was important for them to regain. Further questions relating specifically to the study were also included. The questionnaires were easily understood, and all found them relevant. All questions were answered, and the most important outcome reported was for all functional outcomes, especially the ability to walk properly. Pain was also an important consideration, especially for the internal fixation group. All participants felt that the most important time for measuring outcomes would be after 1 year, but measuring during the first year was also important.

The study protocol was presented to the Patient and Relatives Council at Hospital Lillebaelt afterwards, with no additional remarks.

\section{METHODS: ASSIGNMENT OF INTERVENTIONS Allocation}

Treatment is divided into two strands and patients eligible for inclusion can be enrolled if they provide informed consent. Patients are entered into an electronic database (REDCap) and thereafter randomised using random blocks of $n=4$ or $n=6$ stratified by hospital. When the patient is called to the theatre, the surgeon will determine which implant to use by consulting REDCap; accordingly, REDCap will be used to create two groups representing each implant type.

\section{Blinding}

The surgeon and theatre staff cannot be blinded, but a standard phrase for the surgery will be used to blind the patient. According to standards of care and journal publication requirements, the coordinating staff can reveal the true surgery in case of severe pain or complications; otherwise, patients will not know until the end of the trial. The assessors will assess patients in their own home and will be blinded according to the type of surgery.

\section{Methods}

Data collection, management and analysis.

\section{Data collection methods}

Data will be collected by project staff. Baseline data will be collected during admission, and all data concerning patient-reported outcomes and physical assessment are collected by a physiotherapist in the patient's own home or in the outpatient clinic depending on the participant's wish.

\section{Data management}

Data will be entered directly into the project's REDCap database when assessing or interviewing the participants.

\section{Statistical methods}

All variables are described according to their distribution. Groups will be compared by linear mixed models for numerical data and logistic mixed models for dichotomous data including a random effect for hospital. Both unadjusted analyses as well as analyses adjusting for demographic variables, comorbidity, mobility, type of implant, reoperation and mortality will be carried out. Distributional assumptions on residuals and random effects will be investigated by quantile-quantile plots, and in case of deviations from distributional assumptions bootstrapping with 1000 replicates will be used to estimate CIs and $\mathrm{p}$ values. All group comparisons will be carried out both as intention-to-treat analysis and per-protocol analysis. Mortality will be analysed by Cox-regression with baseline hazards stratified by hospital. As sensitivity analyses competing risk regression models will be applied.

\section{Methods: monitoring \\ Data monitoring}

After data retrieval from the first 70 patients, an interim analysis will be performed regarding mortality and function. If there is a difference of $10 \%$ in 30 days mortality, a consensus decision by steering group whether the trial should be stopped will be conducted. Likewise, if the NMS score shows 2 points or more difference after 3 months, all authors are asked whether the trial should be stopped. This is because the required sample size would then be 66 patients.

\section{Potential harms}

Any unforeseen complications that occur during the trial will be registered in the project's REDCap database.

\section{Auditing}

An adjudication committee will audit all X-ray images.

\section{ETHICS AND DISSEMINATION \\ Research ethics approval}

The study is approved by the Danish Data Protection Agency (19/7429) and the Scientific Ethics Committee with the project ID number S-20180036. It was first approved on the 15th October 2018 and a revision was approved 8th October 2019.

\section{Consent or assent}

The orthopaedic surgeon on call is responsible for including patients. The patients have to give a written consent (online supplemental appendix 1) before entering the trial. 


\section{Confidentiality}

Project data are securely stored in the project's REDCap database, and when the trial is completed, data are stored in the Danish Data Archive.

\section{Ancillary and post-trial care}

Any patients who experience any harm due to this trial will have the same care as all other patients in Denmark through the independent Danish Patient Compensation Association.

\section{Dissemination policy}

This is a national trial and all 20 hospitals providing hip fracture care are included. All relevant healthcare professionals involved in hip fracture treatment in Denmark will, therefore, automatically be informed of the trial results. The results will also be published in international peer-reviewed journals.

\section{Perspective}

By conducting a national pragmatic RCT, external validity will potentially be high. A general problem with trials is the lack of clinical impact afterwards as one paper has shown that it takes an average of 17 years for new findings to reach clinical practice. As this is a nationwide study, the impact of the results is expected to be immediate and high.

\author{
Author affiliations \\ ${ }^{1}$ Orthopaedic Surgery and Traumatology, Lillebaelt Hospital, University Hospital of \\ Southern Denmark, Kolding, Denmark \\ ${ }^{2}$ Orthopaedic Surgery and Traumatology, Odense University Hospital, Odense, \\ Denmark \\ ${ }^{3}$ Orthopaedic Surgery and Traumatology, Aalborg University Hospital, Aalborg, \\ Denmark \\ ${ }^{4}$ Orthopaedic Surgery and Traumatology, Aarhus University Hospital, Aarhus, \\ Denmark \\ ${ }^{5}$ Department of Regional Health Research, University of Southern Denmark, Odense, \\ Denmark \\ ${ }^{6}$ Department of Orthopaedics, Næstved-Slagelse-Ringsted Hospitals, Slagelse, \\ Denmark \\ ${ }^{7}$ Department of Physiotherapy and Occupational Therapy, Næstved-Slagelse- \\ Ringsted Hospitals, Slagelse, Denmark \\ ${ }^{8}$ Orthopaedic Surgery and Traumatology, Bispebjerg Hospital, Kobenhavn, Denmark
}

Acknowledgements Statistics was evaluated by OPEN, Odense Patient data Explorative Network, Odense University Hospital. Graphics was designed by Lise Kryger Simonsen.

Collaborators SENSE collaborators: Thomas Giver Jensen; Mikael Skov Nielsen; Rikke Thorninger; Thomas Egendal; Morten Homilius; Peter Ivan Andersen; Jesper Schønnemann; Michael Krasheninnikoff; Hans-Ulrik Ahler-Toftehøj; Peter Toquer; Tobias Aasvang; Jens Peter Alva-Jørgensen; Michael Mølmer; Sead Hasific; Thomas Brandi Bloch; Lasse Pedersen; Peter Szephalmi; Mohammed Adel Al-Bayati; Frithjof Peitz; Steffan Tábori Jensen; Annie Primdahl.

Contributors BV wrote the first draft of the protocol. SK, HP, MSL, KBH and $\mathrm{OB}$ were invited as the steering committee and the protocol was evaluated and rewritten in collaboration. All collaborators were invited to read, comment and suggest alterations to the protocol. Each of the collaborators is in charge of onsite inclusion and data retrieval.

Funding This work is supported by the Novo Nordisk Foundation (grant number NNF190C0058674), Axel Muusfeldt Foundation (grant number 2019-0265), and A.P. Møller Foundation (grant number 19-L-0088). The trial has also received funds from two public foundations, the Region of Southern Denmark Foundation (grant number 18/51820) and the Hospital Lillebaelt Research Committee (grant number 2019-05).
Disclaimer Neither of the foundations mentioned has any influence on data or reporting.

Competing interests Outside the study, BV has received payment for lectures held for Zimmer Biomet and Osmedic Swemac.

Patient and public involvement Patients and/or the public were involved in the design, or conduct, or reporting, or dissemination plans of this research. Refer to the Methods section for further details.

Patient consent for publication Obtained.

Provenance and peer review Not commissioned; externally peer reviewed.

Open access This is an open access article distributed in accordance with the Creative Commons Attribution Non Commercial (CC BY-NC 4.0) license, which permits others to distribute, remix, adapt, build upon this work non-commercially, and license their derivative works on different terms, provided the original work is properly cited, appropriate credit is given, any changes made indicated, and the use is non-commercial. See: http://creativecommons.org/licenses/by-nc/4.0/.

ORCID iD

Bjarke Viberg http://orcid.org/0000-0001-5169-4282

\section{REFERENCES}

1 National Institute for Health and Care Excellence. Hip fracture: mangement. Clinical guideline [CG124]. National Institute for Health and Care Excellence, 2011.

2 Roberts KC, Brox WT. AAOS clinical practice guideline: management of hip fractures in the elderly. J Am Acad Orthop Surg 2015;23:138-40.

3 Lu Q, Tang G, Zhao X, et al. Hemiarthroplasty versus internal fixation in super-aged patients with undisplaced femoral neck fractures: a 5 -year follow-up of randomized controlled trial. Arch Orthop Trauma Surg 2017;137:27-35.

4 Dolatowski FC, Frihagen F, Bartels S, et al. Screw fixation versus Hemiarthroplasty for Nondisplaced femoral neck fractures in elderly patients: a multicenter randomized controlled trial. J Bone Joint Surg Am 2019;101:136-44.

5 Wamper KE, Sierevelt IN, Poolman RW, et al. The Harris hip score: do ceiling effects limit its usefulness in orthopedics? Acta Orthop 2010;81:703-7.

6 Kristensen PK, Thillemann TM, Søballe K, et al. Are process performance measures associated with clinical outcomes among patients with hip fractures? a population-based cohort study. Int $J$ Qual Health Care 2016;28:698-708.

7 de Morton NA, Harding KE, Taylor NF, et al. Validity of the de Morton mobility index (DEMMI) for measuring the mobility of patients with hip fracture during rehabilitation. Disabil Rehabil 2013;35:325-33.

8 de Morton NA, Berlowitz DJ, Keating JL. A systematic review of mobility instruments and their measurement properties for older acute medical patients. Health Qual Life Outcomes 2008;6:44.

9 Parker MJ, Palmer CR. A new mobility score for predicting mortality after hip fracture. J Bone Joint Surg Br 1993;75:797-8.

10 Kristensen MT, Foss NB, Kehlet H. [Timed Up and Go and New Mobility Score as predictors of function six months after hip fracture]. Ugeskr Laeger 2005;167:3297-300.

11 Kristensen MT, Bandholm T, Foss NB, et al. High inter-tester reliability of the new mobility score in patients with hip fracture. $J$ Rehabil Med 2008;40:589-91.

12 Pedersen TJ, Lauritsen JM. Routine functional assessment for hip fracture patients. Acta Orthop 2016;87:374-9.

13 Sikand M, Wenn R, Moran CG. Mortality following surgery for undisplaced intracapsular hip fractures. Injury 2004;35:1015-9.

14 Bernstein J, Weintraub S, Morris T, et al. Randomized controlled trials for geriatric hip fracture are rare and Underpowered: a systematic review and a call for greater collaboration. J Bone Joint Surg Am 2019;101:e132.

15 Fixation using Alternative Implants for the Treatment of Hip fractures (FAITH) Investigators. Fracture fixation in the operative management of hip fractures (faith): an international, multicentre, randomised controlled trial. Lancet 2017;389:1519-27.

16 Hebert-Davies J, Laflamme G-Y, Rouleau D, et al. Bias towards dementia: are hip fracture trials excluding too many patients? A systematic review. Injury 2012;43:1978-84.

17 Loudon K, Treweek S, Sullivan F, et al. The PRECIS-2 tool: designing trials that are fit for purpose. BMJ 2015;350:h2147.

18 Schmidt M, Pedersen L, Sørensen HT. The Danish civil registration system as a tool in epidemiology. Eur J Epidemiol 2014;29:541-9. 
19 Zwarenstein M, Treweek S, Gagnier JJ, et al. Improving the reporting of pragmatic trials: an extension of the CONSORT statement. BMJ 2008;337:a2390.

20 Chan A-W, Tetzlaff JM, Gøtzsche PC, et al. Spirit 2013 explanation and elaboration: guidance for protocols of clinical trials. BMJ 2013;346:e7586.

21 Garden RS. Low-Angle fixation in fractures of the femoral neck. $J$ Bone Joint Surg Br 1961;43:647-63.

22 Palm H, Gosvig K, Krasheninnikoff M, et al. A new measurement for posterior tilt predicts reoperation in undisplaced femoral neck fractures: 113 consecutive patients treated by internal fixation and followed for 1 year. Acta Orthop 2009;80:303-7.

23 Bech RD, Lauritsen J, Ovesen O, et al. The verbal rating scale is reliable for assessment of postoperative pain in hip fracture patients. Pain Res Treat 2015;2015:1-7.

24 de Morton NA, Davidson M, Keating JL. Validity, responsiveness and the minimal clinically important difference for the de Morton mobility index (DEMMI) in an older acute medical population. BMC Geriatr 2010;10:72.

25 Collin C, Wade DT, Davies S, et al. The Barthel ADL index: a reliability study. Int Disabil Stud 1988;10:61-3.
26 Kristensen MT, Jakobsen TL, Nielsen JW, et al. Cumulated ambulation score to evaluate mobility is feasible in geriatric patients and in patients with hip fracture. Dan Med $J$ 2012;59:A4464.

27 Chammout GK, Mukka SS, Carlsson T, et al. Total hip replacement versus open reduction and internal fixation of displaced femoral neck fractures: a randomized long-term follow-up study. J Bone Joint Surg Am 2012;94:1921-8.

28 Baumgaertner MR, Curtin SL, Lindskog DM, et al. The value of the tip-apex distance in predicting failure of fixation of peritrochanteric fractures of the hip. J Bone Joint Surg Am 1995;77:1058-64.

29 Koldaas MIB, Pedersen JN, Højsager FD, et al. Implant positioning (IMPO) in undisplaced femoral neck fractures: association to reoperation and development of an IMPO scoring system. Injury 2020;51:372-9.

30 Kristensen MT. Hip fractures - Functional assessments and factors influencing in-hospital outcome, a physiotherapeutic perspective. Lund University, 2010.

31 Steihaug OM, Gjesdal CG, Bogen B, et al. Does sarcopenia predict change in mobility after hip fracture? A multicenter observational study with one-year follow-up. BMC Geriatr 2018;18:65. 